\title{
Analytical and numerical time-dependent modelling of SSC blazars variability
}

\section{Stefano Ciprini}

1. Physics Department, University of Perugia, via A. Pascoli, 06123 Perugia, Italy

2. I.N.F.N. Section of Perugia, via A. Pascoli, 06123 Perugia, Italy

3. Tuorla Observatory, Department of Physics and Astronomy, University of Turku, Väisäläntie 20, 21500 Piikkiö, Finland

E-mail: stefano.ciprini@pg.infn.it

\begin{abstract}
A time-dependent synchrotron self-Compton (SSC) leptonic model, developed with some analytical formulations and a numerical code is summarized. This SSC emission model, developed with some analytical formulations and a numerical code, allow to investigate quantitatively the multiwavelength spectral variability of blazars, providing estimates for physical parameters and the bolometric power. The temporal evolution of the electron distribution in a relativistic plasma blob with isotropic magnetic field, is calculated resolving numerically a first order kinetic equation with a fast semi-implicit finite differences scheme derived from a more general solving scheme suitable for diffusion-advection Fokker-Planck equations. The model provides time-dependent synchrotron and inverse Compton emissivities and spectral energy distributions, incorporating radiative cooling as well as light crossing time effects and the relativistic beaming.
\end{abstract}

Workshop on Blazar Variability across the Electromagnetic Spectrum

April 22-25, 2008

Palaiseau, France 


\section{Introduction}

The rapid and large-amplitude variability of blazars implies non-stationary emitting particle distributions, and requires a time-dependent modelling. The energetic and rapid variable radiation can be though as produced in a single active blob into the jet of the blazar, ignited by a shock, and described therefore by a one-zone synchrotron self-Compton (SSC) scenario. Some examples of SSC numerical models are described in: $[8,5,12,10,6,9,13]$. The leptonic SSC and time-dependent model summarized here, is implemented with some analytical calculations and a numerical code, describing the evolution of the relativistic electrons injected with a rate $Q(E)$ $\left[\mathrm{cm}^{-3} \mathrm{~s}^{-1}\right]$ ), in a compact active region (knot, blob) of dimension $R$. The numerical code calculates then the time-dependent synchrotron and inverse Compton (SSC) emissivities and the spectral energy distributions.

\section{Kinetic equation and some solutions}

Stochastic systems like Brownian motion, are characterized by fluctuant interactions, and the macroscopic quantities are non-uniformly distributed resulting in a particle transport. The statistic motion equation of the particle distribution can be approximated by a diffusion equation in case of small fluctuations. The truncation at the second order of the integral operators expansion is the so-called Fokker-Planck (or diffusion-advection) equation [3]. This kinetic equation is used in various fields, and can be profitably used in the simple one-dimensional (1D) form (integrating with spherical coordinates in the momentum space, under an ultra-relativistic regime, and for isotropic and homogeneous distributions) to describe the relativistic electron distribution in the acceleration and cooling regions of a blazar jet. The equation can be written as:

$$
\frac{\partial N(t, E)}{\partial t}=\frac{1}{A(E)} \frac{\partial}{\partial E}\left(B(t, E) N(t, E)+D(t, E) \frac{\partial N(t, E)}{\partial E}\right)-\frac{N(t, E)}{\tau(t, E)}+Q(t, E),
$$

where: 1) $N(t, E) A(E) d E$ is the number of particles per unit volume with energy between $E$ $E+d E$ at time $t$;2) $D(t, E)$ is the diffusion coefficient in the energy space (dispersion around the mean value of the energy gains); 3$) B(t, E)$ is the sum of systematic energy gains (provided by magnetic turbulence interactions, acceleration by shocks, inverse Compton scattering gains, magnetic reconnection, plasma waves, acceleration by electric fields, etc.) and of energy loss terms (synchrotron radiation, Coulomb collisions, adiabatic expansion, etc.); 4) $\tau(t, E)$ is the characteristic escape time or loss rate for cooling; 5) $Q(t, E)$ is the source/injection term; 6) $A(E)$ is the volume element spherically averaged. The nature of the solutions is sensitive to initial and boundary conditions, and on the type of injection $Q$ and losses. Neglecting the escape and losses terms the previous equation assumes the form of a continuity equation: $\partial N / \partial t=\partial F / \partial E$, where $F(t, E)=$ $D(t, E)(\partial N / \partial E)+B(t, E) N$ is the 1D particle flux of particles. The no-flux boundary condition is appropriate in our modelling (particles cannot gain energy without limits, because sources are finite and cooling mechanisms win at high energies). Several methods are able to provide approximated analytical solutions (eigenfunction expansion, WKBJ methods, Laplace transforms, Green Functions, etc.). Green functions are appropriate here, because for diffusion and gain-loss processes the eigenvalue spectrum is continuous. In the "hard-sphere" plasma turbulence approximation results: $D(t, E) \propto E^{2}, B(t, E) \propto E^{2}$ and $\tau(t, E)=\operatorname{cost}([14])$, and here some time-dependent solutions were 
found already by [7]. Considering an instantaneous gain of energetic electrons, a synchrotron and IC cooling, an escape with no diffusive processes $D(t, E)=0$, and expressing all in terms of the non-dimensional Lorentz factor $\gamma=E / m_{e} c^{2}$, the equation for our SSC model is reduced to:

$$
\frac{\partial N(t, \gamma)}{\partial t}=b \frac{\partial}{\partial \gamma}\left(\gamma^{2} N(t, \gamma)\right)+Q(t, \gamma)-\frac{N(t, \gamma)}{\tau(\gamma)} \text {. }
$$

where $-\dot{\gamma}=b \gamma^{2}=\frac{4}{3} \frac{\sigma_{T} c}{m_{e} c^{2}}\left(U_{B}+U_{\text {rad }}(t, \gamma)\right) \gamma^{2}\left[\mathrm{~s}^{-1}\right]$, denoting with $\dot{\gamma}$ the total synchrotron and IC (SSC), cooling rate, and being $U_{B}=B^{2} /(8 \pi)$ and $U_{\text {rad }}(t, \gamma)$ (the photon field energy density). The cooling time-scale is defined by: $t_{\text {cool }}=-\gamma / \dot{\gamma}=\left(m_{e} c^{2}\right) /\left(4 / 3 \sigma_{T} c \gamma\left(U_{B}+U_{\text {rad }}\right)\right)$

If the injection is independent from the time $Q=Q(\gamma)$ and the electrons cools completely before to escape $\left(t_{\text {esc }} \gg t_{\text {cool }}\right)$, the distribution $N$ achieves the equilibrium and results: $N(\gamma)=$ $-1 / \dot{\gamma} \int_{\gamma}^{\gamma_{\max }} d \gamma^{\prime} Q\left(\gamma^{\prime}\right)$. Here if a single-energy injection is assumed (i.e. $\left.Q(\gamma)=Q_{0} \delta\left(\gamma-\gamma_{\max }\right)\right)$ we obtain $N(\gamma) \propto \gamma^{-2}$. In the case of a power law $Q(\gamma)=Q_{0} \gamma^{-p}$, in the interval $[1, \gamma]$ we obtain: $N(\gamma) \propto \gamma^{-(p+1)}$, if $p>1 ; N(\gamma) \propto \frac{1}{\gamma^{2}}\left(\gamma_{\max }^{1-p}-\gamma^{1-p}\right)$ if $p<1$ (and when $\gamma \ll \gamma_{\max }, N(\gamma) \propto \gamma^{-2}$, as in the single-energy case); $N(\gamma) \propto Q_{0} \frac{1}{\gamma^{2}}\left(\ln \gamma_{\max }-\ln \gamma\right)$ if $p=1$. If the injection follows a power law $Q(\gamma)=Q_{0} \gamma^{-p}$, in the interval $\left[\gamma_{\min }, \gamma_{\max }\right]$, and $p>1$, we have a break in the distribution, in correspondence of $\gamma_{\min }: N(\gamma) \propto \gamma^{-2}$ if $\gamma<\gamma_{\text {min }}$ and $N(\gamma) \propto \gamma^{-(p+1)}$ if $\gamma>\gamma_{\text {min }}$.

Solutions of this type are not useful to handle time-dependent spectra and variability. Some analytical solutions to time dependent equations can be found, even if a more complete treatment is possible only with numerical codes. The equation (2.2) can be solved with the method of characteristics and the Green function formalism, in the case of an isotropic pith angle distribution and neglecting the escape term $\left(t_{\text {esc }} \rightarrow \infty\right): \partial N(t, \gamma) / \partial t-\frac{\partial}{\partial \gamma}(B(t, \gamma) N(t, \gamma))=Q(t, \gamma), \quad \Rightarrow \quad \mathscr{L} N(t, \gamma)=$ $Q(t, \gamma)$, with initial value $N(0, \gamma)=f(\gamma)$. The kernel of the equivalent integral equation (Green function), provides a formal solution of our partial differential equation:

$$
N(t, \gamma)=N_{0}(t, \gamma)+\int_{-\infty}^{+\infty} d t^{\prime} \int_{-\infty}^{+\infty} d \gamma^{\prime} G\left(t, \gamma, t^{\prime}, \gamma^{\prime}\right) Q\left(t^{\prime}, \gamma^{\prime}\right)
$$

where $N_{0}(t, \gamma)$, is the general integral of the homogeneous associated equation $\mathscr{L} N_{0}=0$, while the second term is a particular solution of the complete, inhomogeneous equation. Solutions to the initial value homogeneous problem (i.e. a mere $1 \mathrm{D}$ continuity equation, $\partial N_{0} / \partial t+\partial F\left(N_{0}\right) / \partial \gamma=$ $\left.\partial N_{0} / \partial t+\left(d F / d N_{0}\right)\left(\partial N_{0} / \partial \gamma\right)=0\right)$, can be obtained with the methods of the characteristics of Lagrange, reducing it to a system of ordinary differential equations. The used parameterized curve is $\mathscr{C}:\{\gamma=\gamma(r), \gamma(0)=s$, and $\gamma=\gamma(r), \gamma(0)=s\}$, being $r$ the position along $\mathscr{C}$ and $s$ the intersection of the curve with the $\gamma$ axis. The restriction of the function $N_{0}$ to the curve $\mathscr{C}$ implies $d N_{0} / d r=\left(\partial N_{0} / \partial t\right)(d t / d r)+\left(\partial N_{0} / \partial \gamma\right)(d \gamma / d r)$. The solutions of this equation are given by a surface $\mathscr{S}$, produced by the passage of the characteristic curve $\mathscr{C}$, through every point of the initial curve $\Gamma$ defined as $\Gamma=\left\{\left(t, \gamma, N_{0}\right): t=q(s), \gamma=p(s), N_{0}=N_{0}(s), s_{1}<s<s_{2}\right\}$. Solving the characteristic parametric equivalent system, and removing the $r$ and $s$ coordinates, we obtain the solution $N_{0}=f\left(\gamma-\left(d F / d N_{0}\right) t\right)$. In our case $B(t, \gamma)=-\dot{\gamma}=b \gamma^{2}$, and choosing the initial condition $N_{0}(0, \gamma)=f(\gamma)=k \gamma^{-(\alpha+1)}$, we can write the homogeneous solution:

$$
N_{0}(t, \gamma)=k \gamma^{-(\alpha+1)}(1+b \gamma t)^{-(\alpha+1)} \text {. }
$$


Now we search a particular solution of the complete non-homogeneous equation $\mathscr{L} N=Q(t, \gamma)$. The equivalent equation satisfied by the Green function for the differential operator $\mathscr{L}$, is an equation for a point source (Dirac delta): $\mathscr{L} G=\mathbb{I} \Rightarrow \frac{\partial G\left(t, t^{\prime}, \gamma, \gamma^{\prime}\right)}{\partial t}-\frac{\partial}{\partial \gamma}\left(B(t, \gamma) G\left(t, t^{\prime}, \gamma, \gamma^{\prime}\right)\right)=\delta(t-$ $\left.t^{\prime}\right) \delta\left(\gamma-\gamma^{\prime}\right)$, with $B(\gamma)=-\dot{\gamma}=-d \gamma / d t$, and $t=-\int 1 / B(\varepsilon) d \varepsilon=t(\gamma)$. We proceed resolving such integral inverting the relation, and using the found characteristic curves $\gamma(t)=\gamma^{\prime} /\left(1-b \gamma^{\prime} t\right)$, because the injection $Q$ is stationary in this case. Multiply the previous differential equation for the Green function by the function $B(\gamma)$, an ordinary differential equation is obtained. This is solved with a path integral along the characteristic gamma curve, parameterized with the curvilinear coordinate $\gamma=\gamma(t)$ obtaining: $G\left(t, t^{\prime}, \gamma, \gamma^{\prime}\right)=\frac{1}{B\left(\gamma^{\prime}\right)} \int_{-\infty}^{+\infty} d t B(\gamma(t)) \delta\left(t-t^{\prime}\right) \delta\left(\gamma(t)-\gamma^{\prime}\right)$. Remembering that $B(\gamma)=b \gamma^{2}, Q(t, \gamma)=Q_{0} \gamma^{-\alpha}$, and that the $\delta\left(t-t^{\prime}\right)$ implies $t^{\prime}<t$, integrating $G\left(t, t^{\prime}, \gamma, \gamma^{\prime}\right) Q\left(t^{\prime}, \gamma^{\prime}\right)$ in $d t^{\prime}$ and $d \gamma^{\prime}$ we obtain: $\int_{-\infty}^{+\infty} d \gamma^{\prime} B\left(\gamma\left(t^{\prime}\right)\right) \delta\left(\gamma\left(t^{\prime}\right)-\gamma^{\prime}\right) Q\left(\gamma^{\prime}, t^{\prime}\right)=\frac{Q_{0} \gamma^{-(\alpha-2)}}{\gamma^{\prime 2}} \int_{0}^{t} d t^{\prime}\left(1-b \gamma^{\prime} t^{\prime}\right)^{\alpha-2}=$ $\frac{Q_{0} \gamma^{-(\alpha+1)}}{b(\alpha-1)}\left(1-(1-b \gamma t)^{\alpha-1}\right)$. Finally our total solution of the equation (2.2) without escape term is:

$$
N(t, \gamma)=k \gamma^{-(\alpha+1)}(1+b \gamma t)^{-(\alpha+1)}+\frac{Q_{0} \gamma^{-(\alpha+1)}}{b(\alpha-1)}\left(1-(1-b \gamma t)^{\alpha-1}\right) .
$$

When $k=0(N(0, \gamma)=0)$ we have

$$
N(t, \gamma)=\frac{Q_{0} \gamma^{-(\alpha+1)}}{b(\alpha-1)}\left(1-(1-b \gamma t)^{\alpha-1}\right) \simeq\left\{\begin{array}{ll}
Q_{0} \gamma^{-\alpha} t, & \gamma \ll \frac{1}{b t} \\
\frac{Q_{0} \gamma^{-(\alpha+1)}}{b(\alpha-1)}, & \gamma \gg \frac{1}{b t}
\end{array},\right.
$$

where $b=B_{\text {syn }+ \text { com }} / \gamma^{2}=(4 / 3)\left(\sigma_{T} c / m_{e} c^{2}\right)\left(U_{B}+U_{\text {rad }}(t, \gamma)\right)$, corresponding to the solution found by [7] for coupled processes of cooling and injection.

\section{Synchrotron and Synchrotron Self-Compton Spectra}

The synchrotron emissivity of a single particles $j(\omega, E, \alpha)$ [erg s${ }^{-1} \mathrm{sr}^{-1} \mathrm{~Hz}^{-1}$ ], is defined by: $j(\omega, E, \alpha)=\frac{\sqrt{3} e^{3} B}{2 \pi m_{e} c^{2}} \sin \alpha \frac{\omega}{\omega_{c}} \int_{\frac{\omega}{\omega_{c}}}^{\infty} d \eta K_{\frac{5}{3}}(\eta)=\frac{\sqrt{3} e^{3} B}{2 \pi m_{e} c^{2}} \sin \alpha F\left(\frac{\omega}{\omega_{c}}\right)$, where $K_{u}(\eta)$ are the modified Bessel function (hyperbolic Bessel functions) of order $u$, defined with pure imaginary argument, where $F(x)=x \int_{x}^{\infty} d \xi K_{\frac{5}{3}}(\xi)$, and where $\omega_{c}=\frac{3 \gamma^{2} e B}{2 m c} \sin \alpha=\frac{3}{2} \gamma^{2} \omega_{L} \sin \alpha=\frac{3}{2} \gamma^{3} \omega_{B} \sin \alpha$ is the critical pulsation. Supposing a completely random (tangled) magnetic field, we can neglect polarization and use an isotropic emissivity of an electron distribution $J_{i s o}(t, \omega)$, as well as the average on the pitch-angle values $\alpha(\cos \alpha=\mathbf{v} \cdot \mathbf{B} / v B)$, and on the azimuth angle $\varphi$ values, of the total emissivity $J_{\text {hom }}(\omega, \alpha)$, summed on all the polarizations in an homogeneous and isotropic magnetic field $\mathbf{B}$ : $J_{i s o}(t, \omega)=\frac{1}{4 \pi} \int_{0}^{2 \pi} d \varphi \int_{0}^{\pi} d \alpha \sin \alpha\left(\frac{1}{4 \pi} \int_{0}^{\infty} d E N j_{i s o}(\omega, E, \alpha)\right)=\frac{\sqrt{3} e^{3} B}{2 \pi m_{e} c^{2}} \frac{1}{4 \pi} \int_{0}^{\infty} d E N\left(\frac{1}{2} \int_{0}^{\pi} d \alpha \sin ^{2} \alpha F\left(\frac{2 \sigma}{\sin \alpha}\right)\right)$, where $N=N(t, E), \omega_{L}=(e B) /(m c)$ is the Landau pulsation, $\omega_{B}=\omega_{L} / \gamma$ the cyclotron pulsation, and $\varpi=\omega /\left(3 \omega_{L} \gamma^{2}\right)$ the normalized pulsation. This not trivial integral can be handled in several steps: first integrating by parts in $d \alpha$; using then a variable substitution $\cos \alpha=\sinh \beta / \cosh \beta$; then solving in the complex domain the integrals containing the $K_{u}(\eta)$ Bessel functions (i.e. $\int_{0}^{\infty} d x \cosh (2 v x) K_{2 u}(2 a \cosh x)=\frac{1}{2} K_{v+u}(a) K_{v-u}(a)$ and $\left.\int_{0}^{\infty} d x K_{v \pm u}(2 a \cosh x) \cosh [(v \mp u) x]=\frac{1}{2} K_{v}(a) K_{u}(a)\right) ;$ 

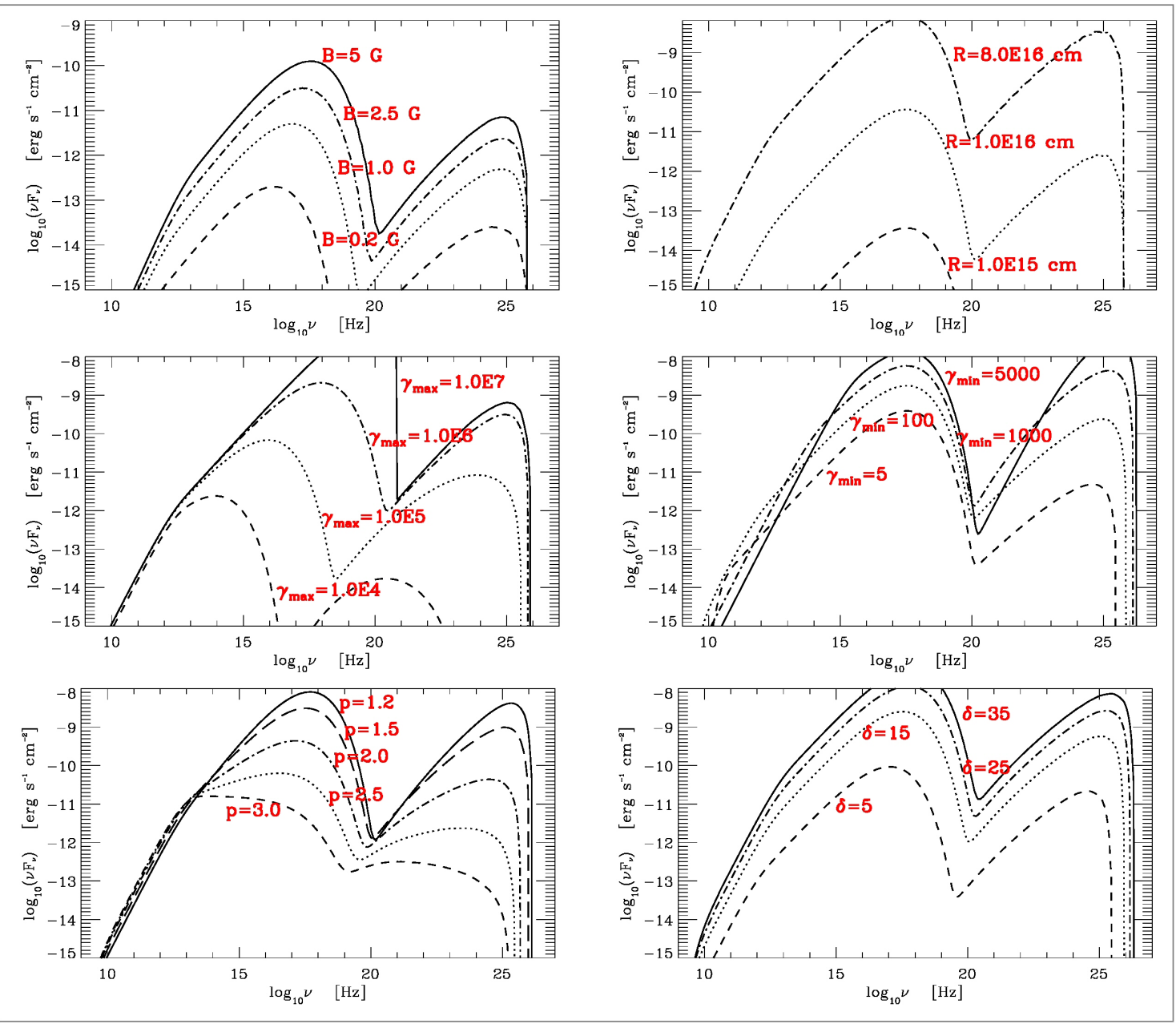

Figure 1: Synchrotron self-Compton (SSC) spectral energy distributions (SEDs) produced by the numerical code for different changes of parameters (magnetic field intensity $B$, region size $R$, maximum Lorentz factor of the injected electron distribution $\gamma_{\max }$, minimum Lorentz factor of the injected electron distribution $\gamma_{\min }$, energy index of the power law electron distribution injected $p$, bulk Doppler factor of beaming $\mathscr{D}$ ).

and using algebraic properties of the $K_{u}(\eta)$ functions, i.e. $K_{u-1}(x)-K_{u+1}(x)=-(2 u / x) K_{u}(x)$. After the calculations the resulting single electron emissivity averaged on the pitch angles, to be convolved with the electron energy distribution $N$ in the isotropic case is:

$$
j_{i s o}(\omega, \gamma)=\frac{3 \sqrt{3} \sigma_{T} c U_{B}}{\pi \omega_{L}} \varpi^{2}\left(K_{\frac{4}{3}}(\varpi) K_{\frac{1}{3}}(\varpi)-\frac{3}{5} \varpi\left(K_{\frac{4}{3}}^{2}(\varpi)-K_{\frac{1}{3}}^{2}(\varpi)\right)\right),
$$

measured in [erg s${ }^{-1} \mathrm{sr}^{-1} \mathrm{~Hz}^{-1}$ ]. In our SSC modelling the power law injection $Q$ is defined in a finite $\left(\gamma_{\min }, \gamma_{\max }\right)$ range, thus: $J_{s y n}(t, \omega)=1 /(4 \pi) \int_{\gamma_{\min }}^{\gamma_{\max }} d \gamma N(t, \gamma) j_{i s o}(\omega, \gamma)$, and the time dependent absorption coefficient is: $k(t, \omega)=\pi /\left(m_{e} \omega\right) \int_{1}^{\infty} d \gamma \frac{N(t, \gamma)}{\gamma p} \frac{\partial}{\partial \gamma}\left(\gamma p j_{i s o}(\omega, \gamma)\right) \quad\left[\mathrm{cm}^{-1}\right]$, with $p=m_{e} c \sqrt{\gamma^{2}-1}$. For a region of dimension $R$ along the line of sight, the optical depth is $\tau(t, \omega)=$ $k(t, \omega) R$, and the self-absorption frequency in which $\tau\left(v_{a}\right)=1$ can be derived, while the intensity is the solution of the radiative transfer equation: $I_{s y n}(t, \omega)=J_{s y n}(t, \omega) / k(t, \omega)\left(1-e^{-k(t, \omega) R}\right)$, $\left[\mathrm{erg} \mathrm{s}^{-1} \mathrm{~cm}^{-2} \mathrm{sr}^{-1} \mathrm{~Hz}^{-1}\right.$. 

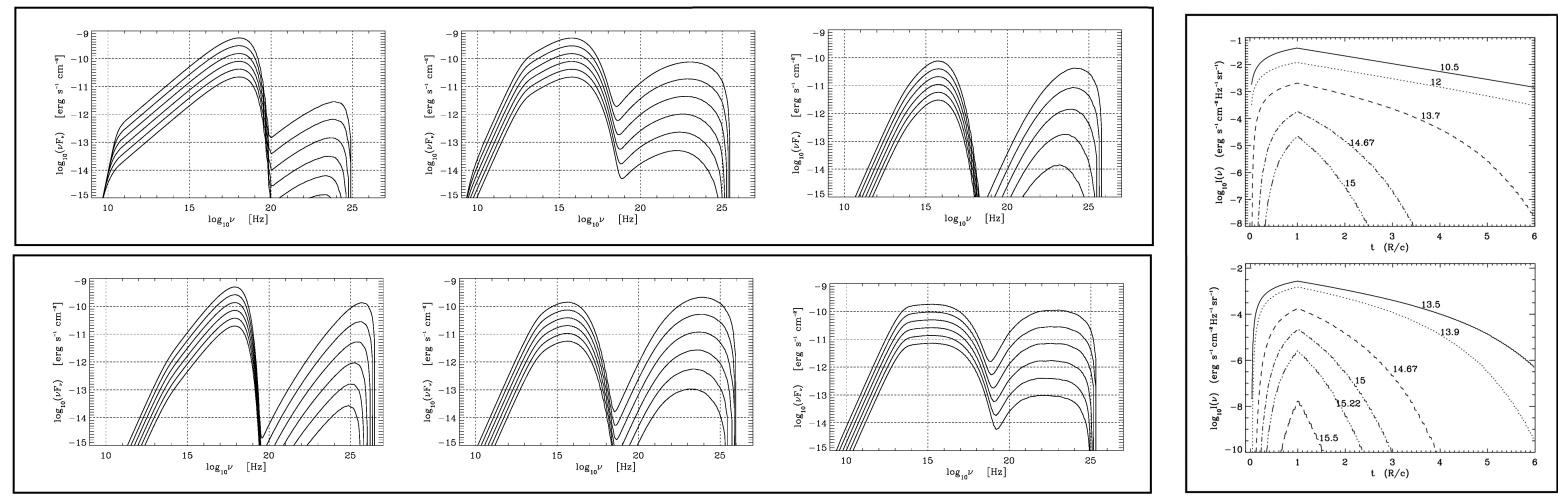

Figure 2: Left panels: Examples of numerical simulations of time-dependent SED evolution, produced with the SSC code, after the injection of high energy electrons, as seen in the observer frame. The different SED shapes are obtained using different combinations of the physical parameters. In all the simulations only the redshift $z=0.5$ and single power-law injection exponentially dumped $\gamma^{-p} e^{c_{0} \gamma / \gamma_{\max }}$ are used. Continuous lines represent temporal stages at multiples of $t_{\text {cross }}=R / c$. Right panels: Simulation of light curves (time in unit of $t_{\text {cross }}$ ) at different synchrotron frequencies (indicated in $\log _{10}(v)$ ). In these plots the crossing time convolution is not taken into account, and thus the curves do not display a plateau.
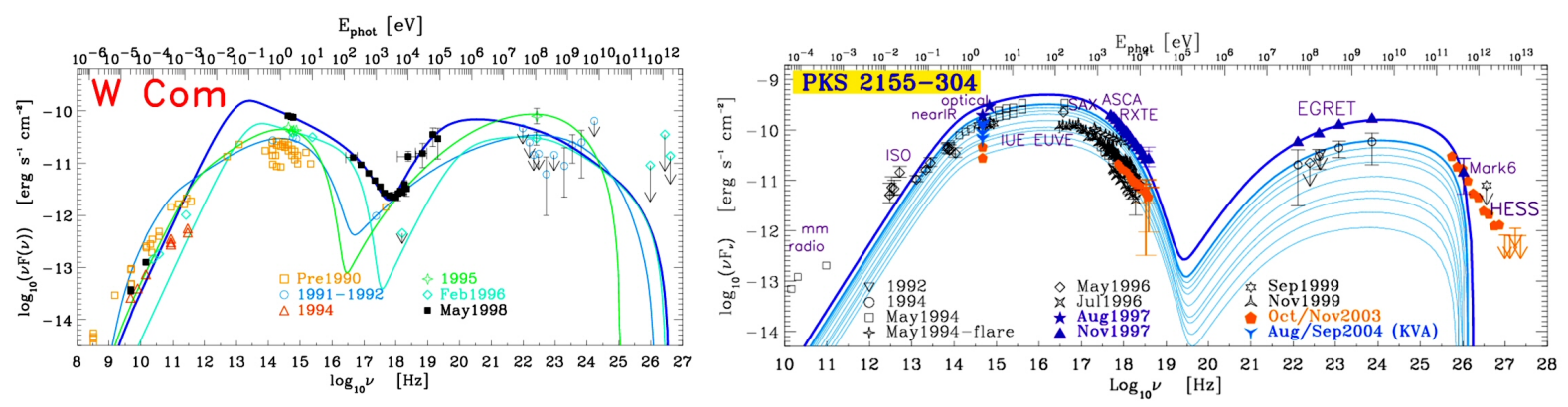

Figure 3: Left panel: SEDs of W Com (ON 231, B2 1219+28; $z=0.102)$ and SSC model fit attempts. The quasi-simultaneous May 1998 data show an interesting broken (two components) X-ray spectrum observed by Beppo-SAX. Further 3 models for quasi-simultaneous and not-simultaneous older data of W Com are also reported. Right panel: SED of PKS 2155-304 (1ES 2155-304; $z=0.116$ ) assembled with observations available around mid-90s. The solid lines indicate one SSC model fit attempt, and the post-flare cooling evolution of the November 1997 outburst. High-energy detections in this epoch at X-rays and at $\gamma$-ray bands by EGRET, Mark6 are reported. HESS data obtained in Oct.-Nov.2003 are also added for comparison [1].

The inverse Compton (IC) spectrum is calculated as pure SSC (interaction of electrons with the isotropic synchrotron photon distribution) integrating the single electron scattering spectrum over the electron distribution $N(t, \gamma)[2]: J_{c o m}^{(S S C)}\left(t, v_{f}\right)=\int_{v_{s}^{\min }}^{v_{s}^{\max }} d v_{s} \int_{\gamma_{1}}^{\gamma_{2}} d \gamma N(t, \gamma) j_{c o m}\left(\gamma, v_{s}, v_{f}\right) I_{s y n}\left(v_{s}\right)$, $\left[\mathrm{erg} \mathrm{s}{ }^{-1} \mathrm{~cm}^{-3} \mathrm{sr}^{-1} \mathrm{~Hz}^{-1}\right.$, where: $v_{s}$ is the frequency of the incident synchrotron radiation; $v_{f}$ is the frequency of the outgoing radiation, after the IC scattering; $N(\gamma)$ is the electron distribution in the emitting region; $j_{c o m}\left(\gamma, v_{s}, v_{f}\right)$ is the IC emissivity of the single electron,; $I_{s y n}\left(v_{s}\right)$ is the intensity of the incident synchrotron radiation; and $\gamma_{1}=\max \left[\sqrt{\frac{v_{f}}{4 v_{s}}}, \gamma_{\min }\right], \gamma_{2}=\min \left[\gamma_{\max }, \frac{3 m_{e} c^{2}}{4 h v_{s}}\right]$. There the inverse Compton emissivity of the single electron, (scattering the monochromatic radiation of frequency $\left.v_{s}\right)$, is defined as $[2,15]:\left(N(\gamma) \sigma_{T} F_{0}\right) /\left(4 \gamma^{2} \beta^{2} \varepsilon_{s}\right) f\left(\varepsilon_{s}, \varepsilon_{f}\right)$, with $f\left(\varepsilon_{s}, \varepsilon_{f}\right)=(1+\beta) \frac{\varepsilon_{f}}{\varepsilon_{s}}-$ $(1-\beta)$ when $\frac{1-\beta}{1+\beta} \leqslant \frac{\varepsilon_{f}}{\varepsilon_{s}} \leqslant 1$; with $f\left(\varepsilon_{s}, \varepsilon_{f}\right)=(1+\beta)-\frac{\varepsilon_{f}}{\varepsilon_{s}}(1-\beta)$ when $1 \leqslant \frac{\varepsilon_{f}}{\varepsilon_{s}} \leqslant \frac{1+\beta}{1-\beta}$ and with $f\left(\varepsilon_{s}, \varepsilon_{f}\right)=0$ elsewhere (being $\varepsilon=h v$ and $\beta=v / c$ ). When the region of dimension $R$ is fully transparent to the IC frequencies, we can set $I_{c o m}\left(t, v_{f}\right)=J_{c o m}\left(t, v_{f}\right) R$. 
The total spectrum of the synchrotron and the inverse Comtpon radiation is: $I_{t o t}(t, v)=$ $I_{s y n}(t, v)+I_{c o m}(t, v)$ and the luminosity (bolometric power) $L(t, v)=\left(16 \pi^{2} / 3\right) R^{2} I_{t o t}(t, v)$. The observed quantities corrected for the relativistic beaming and cosmological effects are: $v_{o b s}=\mathscr{D} v$, $L_{o b s}\left(t, v_{o b s}\right)=(\mathscr{D} /(1+z))^{3} L(t, v)$, where $\mathscr{D}$ is the bulk Doppler beaming factor.

\section{The numerical implementation}

The algorithm for the solution of the time-dependent kinetic equation consists of a direct method resolving the equivalent system of algebraic discretized equations obtained with a one step finite difference scheme. We used the fast and robust semi-implicit conservative scheme of [4], improved by [11]. This numerical method is the best compromise between functionality, stability and accuracy. Intervals $\Delta t$ and $\Delta \gamma$ of the two-dimensional mesh representing the electron number density function $N(t, \gamma)$, can be rather broad, and the semi-implicit method provides accurate time-dependent solutions (and non-negativity of solutions). The energy ( $\gamma$ factor) has a logarithmic equally spaced mesh, while the time a linear mesh. After the solving of the equation, at every discrete time step the synchrotron emissivity and intensity, the IC (SSC) intensity, and the total intensity and luminosity are calculated based on the analytical formulations summarized in the previous sections. Beaming effects are then evaluated as well. Examples of some SEDs produced by our SSC code are reported in Fig.1, 2 and 3.

\section{References}

[1] Aharonian F., Akhperjanian A.G. , Bazer-Bachi A.R. et al. 2005, A\&A, 442, 895

[2] Blumenthal G.R., \& Gould R.J. 1970, Rev. Mod. Phys., 42, 237

[3] Chandrasekhar S. 1943, Rev. Mod. Phys., 15, 1

[4] Chang J.S., \& Cooper G. 1970, Journ. Comput. Phys., 6, 1

[5] Chiaberge M., \& Ghisellini G. 1999, MNRAS, 306, 551

[6] Chiang J. \& Böttcher M. 2002, ApJ, 564, 92

[7] Kardashev N.M. 1962, Soviet Astronomy-AJ, 6, 317

[8] Kirk J.G., Rieger F.M., \& Mastichiadis A. 1998, A\&A, 333, 452

[9] Krawczynski H., Coppi P.S., \& Aharonian F. 2002, MNRAS, 336, 721

[10] Kusunose M., Takahara F., \& Li, H. 2000, ApJ, 536, 299

[11] Larsen, E.W., Levermore, C.D., Pomraning, G.C., \& Sanderson, J.G. 1985, Journ. Comput. Phys., 61,359

[12] Li H., \& Kusunose M. 2000, ApJ, 536, 729

[13] Moderski R., Sikora M., \& Błażejowski M. 2003, A\&A, 406, 855

[14] Parker E.N., \& Tidman D.A. 1958, Phys. Rev., 111, 1206

[15] Rybicki G.B., \& Lightman A.P., Radiative Processes in Astrophysics, 1979, New York: Wiley Interscience

[16] Tagliaferri G., Ghisellini G., Giommi P. et al., 2000, A\&A 354, 431 